\title{
COMPLETENESS IN THE SEQUENTIAL CASE
}

\author{
By E. L. Lehmann and Charles Stein \\ University of California, Berkeley
}

1. Summary. Recently, in a series of papers, Girshick, Mosteller, Savage and Wolfowitz have considered the uniqueness of unbiased estimates depending only on an appropriate sufficient statistic for sequential sampling schemes of binomial variables. A complete solution was obtained under the restriction to bounded estimates. This work, which has immediate consequences with respect to the existence of unbiased estimates with uniformly minimum variance, is extended here in two directions. A general necessary condition for uniqueness is found, and this is applied to obtain a complete solution of the uniqueness problem when the random variables have a Poisson or rectangular distribution. Necessary and sufficient conditions are also found in the binomial case without the restriction to bounded estimates. This permits the statement of a somewhat stronger optimum property for the estimates, and is applicable to the estimation of unbounded functions of the unknown probability.

2. Introduction. The notions of completeness and bounded completeness of a family of distributions were introduced in $[1,2]$ in connection with the problems of similar regions and unbiased estimation. The question of whether either of these two properties pertains to various families of distributions that are of interest in statistics was discussed in [2] under the assumption of fixed sample size. The only sequential problems of this kind that have been treated in the literature (with quite different terminology) refer to the binomial case. For this case Girshick, Mosteller and Savage [3] found necessary (and also certain sufficient) conditions on the sequential sampling scheme for completeness, while Wolfowitz [4] and Savage [5] gave necessary and sufficient conditions for bounded completeness.

If $T$ is a random variable distributed over an additive class of sets in some space according to a distribution $P_{\theta}^{T}$ with $\theta$ in some set $\omega$, then the family $\mathscr{P}^{T}=\left\{P_{\theta}^{T} \mid \theta \in \omega\right\}$ of possible distributions of $T$ is said to be complete if

$$
\int f(t) d P_{\theta}^{T}(t)=0, \quad \text { for all } \theta \epsilon \omega,
$$

implies

$$
f(t)=0, \quad \text { a.e. } \mathscr{P}^{T},
$$

that is, for all $t$ except possibly in a set $N$ for which $P_{\theta}^{T}(N)=0$ for all $\theta \epsilon \omega$. The family $\mathscr{P}^{T}$ is said to be boundedly complete if this implication holds under the assumption that $f$ is bounded.

The relation of these concepts to the problem of unbiased estimation is an 
immediate consequence of a theorem of Blackwell [6]. Let $X$ be a random variable with distribution $P_{\theta}^{X}, \theta \epsilon \omega$, and let $T$ be a sufficient statistic for $\theta$. Denote by $P_{\theta}^{T}$ the distribution of $T$, and suppose that $\mathcal{P}^{T}$ is complete. Then every function $g(\theta)$ for which there exists an unbiased estimate, that is, a function $\phi$ such that

$$
E_{\theta} \phi(X)=g(\theta), \quad \text { for all } \theta \epsilon \omega,
$$

possesses an unbiased estimate with uniformly minimum variance. One can say furthermore that if $\phi(X)$ is any unbiased or bounded unbiased estimate of $g(\theta)$, then the optimum estimate guaranteed by the above statements is the condifional espectation of $\phi(X)$ given $T$.

The aim of the present paper is to obtain certain results concerning completeness in sequential sampling schemes. Some necessary conditions for completeness are given in section 3 , and these are used to obtain necessary and sufficient conditions for completeness when the random variable being sampled has a Poisson or rectangular distribution. In section 4 it is shown that certain necessary conditions given in [3] for the binomial case are also sufficient.

3. A necessary condition for completeness. The sequential sampling schemes with which we are concerned are of the following nature. There is given a sequence of real valued random variables $X_{1}, X_{2}, \cdots$ with a joint distribution depending on a real parameter $\theta$, which ranges over a set $\omega$. We shall assume that for each $m$ the set of variables $X_{1}, \cdots, X_{m}$ admits a real valued sufficient statistic $T_{m}=t_{m}\left(X_{1}, \cdots, X_{m}\right)$ for $\theta$, and that for each $m$ the family $\mathscr{P}^{T_{m}}$ of distributions of $T_{m}$ is complete. We next suppose that there is given a stopping rule, which is such that after $m$ observations have been taken, the decision of whether or not to take an $m+1 s t$ observation depends only on the value of $t_{m}\left(X_{1}, \cdots, X_{m}\right)$. It follows (see [6]) that if the total number of observations is $n$ (a random variable which may be infinite), then $\left(T_{n}, n\right)$ is a sufficient statistic for $\theta$. We shall say that the sequential procedure is complete if the family of distributions of $\left(T_{n}, n\right)$ is complete. Throughout, we shall assume that all sequential procedures in question are closed, i.e. that for each $\theta \epsilon \omega, n$ is finite with probability 1 .

Let $Y$ be a random variable distributed over a Euclidean space according to a distribution $P_{\theta}^{Y}$ with $\theta$ in $\omega$. We shall say that a point $y$ lies in the positive sample space of $Y$ if there exists $\theta \epsilon \omega$ such that every open set containing $y$ has positive probability for this $\theta$, and that $y$ is an impossible point if it lies in the complement of the positive sample space. Consider now a sequential sampling scheme as described above. For any integers $m<p$ we shall denote by $W_{p}^{m}$ the positive sample space of $T_{p}$ given the first $m$ steps of the stopping rule, that is, given for $i=1, \cdots, m$ the set $S_{i}$ of values of $T_{i}$ for which sampling is discontinued after the $i$ th observation. Since all the $T$ 's are real valued, the sets $W_{p}^{m}$ are sets of real numbers satisfying the obvious condition $W_{p}^{m-1} \geqq W_{p}^{m}$. The union $U S_{m}\left(S_{m}\right.$ is the set of points of $W_{m}^{m-1}$ for which no $m+1$ st observation is 
taken) will be called the set of stopping or boundary points, the points belonging to some $W_{m}^{m-1}-S_{m}$ are the continuation points.

We need the following

Lemma 1. A necessary condition for a sequential procedure of the type described above to be complete is that every procedure obtained from the given one by truncation be complete. ${ }^{1}$

This is an immediate consequence of the following more general

Lemma 2. Let $X_{1}, X_{2}, \cdots$ be as before a sequence of random variables such that for each $m$ the set $X_{1}, \cdots, X_{m}$ admits a real valued sufficient statistic $T_{m}=t_{m}\left(X_{1}, \cdots, X_{m}\right)$. Let $\Sigma_{1}, \Sigma_{2}, \cdots, \Sigma_{r}$ each be a complete, closed, sequential procedure based on these sufficient statistics. Let $\Sigma_{1} \cup \Sigma_{2} \cup \cdots \cup \Sigma_{r}$ denote the sequential procedure according to which we continue taking observations until at least one of the stopping rules $\Sigma_{1}, \cdots, \Sigma_{r}$ tells us to stop. Then the procedure $\Sigma_{1} \cup \cdots \cup \Sigma_{r}$ is complete.

This clearly implies Lemma 1 . For if one takes for $\Sigma_{1}$ any closed, complete sequential procedure and for $\Sigma_{2}$ a procedure of fixed sample size, then $\Sigma_{1} u \Sigma_{2}$ is the associated truncated procedure.

Proof of Lemma 2. It is sufficient to prove the result for the case $r=2$.

Let $n_{1}, n_{2}, n$ denote the number of observations taken under $\Sigma_{1}, \Sigma_{2}, \Sigma_{1} \cup \Sigma_{2}$ respectively. Then $n=n_{1}$ if $n_{1} \leqq n_{2}, n=n_{2}$ if $n_{1} \geqq n_{2}$. Let $f$ be any function on $\Sigma_{1} \cup \Sigma_{2}$ such that

$$
E_{\theta} f\left(T_{n}, n\right)=0 \text { for all } \theta \epsilon \omega
$$

Then

$$
\left.\begin{array}{l}
E_{\theta} E\left[f\left(T_{n}, n\right) \mid T_{n_{1}}, n_{1}\right]=0 \\
E_{\theta} E\left[f\left(T_{n}, n\right) \mid T_{n_{2}}, n_{2}\right]=0
\end{array}\right\} \text { for all } \theta \epsilon \omega .
$$

Since $\Sigma_{1}$ and $\Sigma_{2}$ are complete it follows that

$E\left[f\left(T_{n}, n\right) \mid T_{n_{1}}=t_{1}, n_{1}=\gamma_{1}\right]=E\left[f\left(T_{n}, n\right) \mid T_{n_{2}}=t_{2}, n_{2}=\gamma_{2}\right]=0$, a.e.

Hence

$$
\begin{aligned}
0 & =P\left(n_{1} \leqq n_{2} \mid T_{n_{1}}=t_{1}, n_{1}=\gamma_{1}\right) f\left(t_{1}, \gamma_{1}\right) \\
& +P\left(n_{1}>n_{2} \mid T_{n_{1}}=t_{1}, n_{1}=\gamma_{1}\right) E\left[f\left(T_{n_{2}}, n_{2}\right) \mid T_{n_{1}}=t_{1}, n_{1}=\gamma_{1}, n_{1}>n_{2}\right],
\end{aligned}
$$

and the analogous condition holds with the subscripts 1 and 2 interchanged.

We shall prove that $f\left(T_{n}, n\right)=0$, a.e., by induction over the possible values of $n$. Suppose, therefore, that for some integer $m$

$$
P_{\theta}\left(n \leqq m, f\left(T_{n}^{\prime}, n\right) \neq 0\right)=0 .
$$

(This is certainly true for $m=0$.) It then follows that if we take $\gamma_{1}=m+1$ in (3) the second term of the right hand side vanishes, so that

$$
0=P\left(n=n_{1} \mid T_{n_{1}}=t_{1}, n_{1}=m+1\right) f\left(t_{1}, m+1\right) .
$$

${ }_{1}^{1}$ The authors would like to thank Mr. E. Fay for pointing out an error in the original proof of this Lemma. 
Hence,

$$
\begin{aligned}
P_{\theta}\left(n=n_{1}\right. & \left.=m+1, f\left(T_{n_{1}}, n_{1}\right) \neq 0\right) \\
& \leqq P_{\theta}\left(n=n_{1}=m+1, P\left(n=n_{1} \mid T_{n_{1}}, n_{1}\right)=0\right)=0 .
\end{aligned}
$$

Analogously we see that

$$
P_{\theta}\left(n=n_{2}=m+1, f\left(T_{n_{2}}, n_{2}\right) \neq 0\right)=0
$$

and, adding, that

$$
P_{\theta}\left(n=m+1, f\left(T_{n}, n\right) \neq 0\right)=0 .
$$

This completes the induction.

We need further the notion of strong completeness. Consider a random variable $W=(U, V)$, suppose that the distribution of $W$ depends on $\theta$, and that $U$ is a sufficient statistic for $\theta$. Let $P_{u}^{v}$ be the conditional distribution of $V$ given $U=u$-this is independent of $\theta$ since $U$ is a sufficient statistic for $\theta$-and let $\mathscr{P}^{V^{*}}=\left\{\mathscr{P}_{u}^{V}\right\}$. We say that the pair $\mathscr{P}^{W}, \mathscr{P}^{V^{*}}$ is strongly complete if the conditions (i) $E_{\theta} f(V)$ exists for all $\theta$,

(ii) $E(f(V) \mid U=u)=0$ for almost all $u$, imply

$$
f(v)=0, \quad \text { a.e. } \mathscr{P}^{v} \text {. }
$$

For brevity, we shall then usually say that $\left\{\mathscr{P}_{u}^{v}\right\}$ is strongly complete.

We can now state the following necessary condition for completeness.

Theorem. If a closed sequential procedure of the type considered above is complete, then

(i) $S_{m}$ is almost empty for every $m$ for which $W_{m+1}^{m-1}-W_{m+1}^{m}$ is almost empty,

(ii) for each $m$ for which $S_{m}$ is not almost empty, the family of conditional distributions of $T_{m}$ given $T_{m+1}=t$ (as t ranges over $W_{m+1}^{m-1}-W_{m+1}^{m}$ ) is strongly complete.

Proof. For any $t \in W_{m+1}^{m-1}-W_{m+1}^{m}$ the positive sample space of $T_{m}$ given $T_{m+1}=t$ is clearly contained in $S_{m}$. Suppose first that (ii) is violated and consider the sequential procedure obtained from the given one by truncation after $m+1$ observations. By the lemma it will be enough to show that the truncated procedure is not complete. For this purpose let us assume that regardless of the stopping rule all $m+1$ variables $X_{1}, \cdots, X_{m+1}$ are observed. We want to construct an estimate of zero based on the sufficient statistic for the truncated procedure. This estimate must be a function of $T_{1}$ for $T_{1} \epsilon S_{1}$, of $T_{2}$ for $T_{2} \epsilon S_{2}$, etc. That is, although we may imagine that the full sample of size $m+1$ is taken, we must be careful not to use observations that are impossible when the stopping rule is followed.

We shall now show that there exists an unbiased estimate of zero which is zero over $S_{1}, \cdots, S_{m-1}$, equal to $f\left(T_{m}\right)$ on $S_{m}$ and $g\left(T_{m+1}\right)$ on $W_{m+1}^{m}$ where $f$ and $g$ will be defined below. Since expectation equals expectation of conditional expectation, a statistic is an unbiased estimate of zero if its expectation exists 
and its conditional expectation given $T_{m+1}=t$ is zero for almost all $t$. In our case this condition is equivalent to

$$
\int_{S_{m}} f(u) d P_{m}\left(u \mid T_{m+1}=t\right)+g(t) \int_{W^{m-1}-S_{m}} d P_{m}\left(u \mid T_{m+1}=t\right)=0
$$

for almost all $t \in W_{m+1}^{m}$,

$$
\int_{S_{m}} f(u) d P_{m}\left(u \mid T_{m+1}=t\right)=0
$$

for almost all $t \notin W_{m+1}^{m}$, i.e. for almost all $t \in W_{m+1}^{m-1}-W_{m+1}^{m}$, since $t W_{m+1}^{m-1}$ implies $P\left(S_{m} \mid T_{m+1}=t\right)=0$,

together with the existence of $E_{\theta}\left(f\left(T_{m}\right) \mid n=m\right)$ and $E_{\theta}\left(g\left(T_{m+1}\right) \mid n=m+1\right)$. Since (ii) does not hold there exists $f$ not vanishing a.e. such that $E_{\theta}\left(f\left(T_{m}\right) \mid n=m\right)$ exists and (5) is satisfied. If $g$ is defined by (4), $E_{\theta}\left(g\left(T_{m+1}\right) \mid n=m+1\right)$ exists, and this completes the proof of the necessity of (ii).

The necessity of (i) is now obvious. For if (i) is violated, then (5) is satisfied vacuously, and we can take $f$ to be an arbitrary positive valued function (for example) and (4) will then be satisfied.

As immediate consequences of this theorem we shall obtain two conditions, which are easier to apply than condition (ii).

Corollary 1. A necessary condition for completeness is that for no $m$ there exists a subset $A$ of $S_{m}$ such that

$$
P_{\theta}(A)>0 \text { for some } \theta
$$

and

$$
P\left(A \mid T_{m+1}=t\right)=0 \text { for almost all } t \in W_{m+1}^{m-1}-W_{m+1}^{m} .
$$

Corollary 2. Suppose that the sequence of $X$ 's is such that in the non-sequential case for all $m, p$ with $m<p$ the positive sample space of $T_{m}$ given $T_{p}=t$ is the intersection of the unconditional positive sample space of $T_{m}$ with the interval $[0, t]$. Then a necessary condition for a sequential procedure to be complete is that each $S_{m}$ differ from a half-open interval (possibly empty) $\left[a_{m}, b_{m}\right)$ with $a_{m} \leqq b_{m}, a_{1}=0$, $a_{m+1}=b_{m}$, by a set of probability 0 .

Proof. Let $r$ be the first value of $m$ for which this condition is not satisfied. Then there exists $c>b_{r-1}$ such that the sets $S_{r} \cap[c, \infty)$ and $\bar{S}_{r} \cap\left[b_{r-1}, c\right)$ both have positive probability. The result now follows from Corollary 1 if one puts $A=S_{r} \cap[c, \infty)$.

Next we consider some examples.

EXAMPLE 1. Let $X_{1}, X_{2}, \cdots$ be independently normally distributed with known variance and unknown mean $\theta$. In this case $T_{m}=\sum_{i=1}^{m} X_{i}$, and since the positive sample space of $T_{m+1}$ is the infinite interval regardless of the values of $T_{1}, \cdots, T_{m}$ it follows from condition (i) of the theorem that no sequential procedure is complete, with the trivial exception of the procedures with fixed sample size. 
Example 2. Let $X_{1}, X_{2}, \cdots$ be independently uniformly distributed over the interval $(0, \theta), 0<\theta<\infty$. Then $T_{m}=\max \left(X_{1}, \cdots, X_{m}\right)$ and Corollary 2 gives a necessary condition for completeness. If the procedure is truncated we can deduce sufficiency of this condition from (5). However, this proof does not apply to the general case. The following proof of sufficiency is similar to some of the proofs in $[3,4,5]$.

Suppose $S_{1}, S_{2}, \cdots$ form a set of adjoining intervals (some of them possibly empty), $S_{m}=\left[a_{m}, b_{m}\right)$, and suppose there is a non-zero unbiased estimate of zero, $\Phi=\phi\left(T_{n}, n\right)$. Let $m$ be the smallest integer for which $\phi$ is not zero almost everywhere on $S_{m}$. Then

$$
E_{\theta}(\Phi)=P_{\theta}(n=m) E_{\theta}(\Phi \mid n=m)+\sum_{j=m+1}^{\infty} P_{\theta}(n=j) E_{\theta}(\Phi \mid n=j) \stackrel{(\theta)}{\equiv 0,}
$$

and hence

$$
P_{\theta}(n=m) E_{\theta}(\Phi \mid n=m) \stackrel{(\theta)}{\equiv}-\sum_{j=m+1}^{\infty} P_{\theta}(n=j) E_{\theta}(\Phi \mid n=j) .
$$

Now the right hand side of (6) is zero when $\theta \leqq b_{m}$, since it is then impossible that $T_{j} \in S_{j}$ for any $j>m$. Hence

$$
E_{\theta}\left[\phi\left(T_{m}, m\right) \mid a_{m} \leqq T_{m}<b_{m}\right]=0 \text { for all } \theta \leqq b_{m},
$$

and therefore

$$
\int_{a_{m}}^{\theta} \phi(x, m) x^{m-1} d x=0 \text { for all } \theta \text { in }\left[a_{m}, b_{m}\right] .
$$

But this implies $\phi(x, m)=0$ almost everywhere in $S_{m}$, which is a contradiction.

Example 3. Let $X_{1}, X_{2}, \cdots$ be independently distributed according to a Poisson distribution with mean $\theta$. Then $T_{m}=\sum_{i=1}^{m} X_{i}$ and again we can apply Corollary 2. To prove sufficiency we proceed as in example 2. If the condition of Corollary 2 is satisfied we may write without ambiguity $\psi\left(T_{n}\right)$ for $\phi\left(T_{n}, n\right)$. Let $c$ be the smallest value of $T_{n}$ for which $\underset{(\theta)}{\psi\left(T_{n}\right)} \neq 0$. Then if the probability of $T_{n}=j$ is $k(j) \theta^{j} e^{-\theta m_{j}}$, the identity $E_{\theta}(\Phi) \stackrel{(\theta)}{\equiv} 0$ implies

$$
\phi(c) k(c) \theta^{c} \rho \stackrel{(\theta)}{\equiv} \sum_{j=c+1}^{\infty} \phi(j) k(j) \phi^{j} \cdot \varphi(c) k(c) \theta^{c} e^{-\theta m_{c}} \equiv \sum_{j=c+1}^{\infty} \varphi(j) k(j) \theta^{j} e^{-\theta m_{j}} .
$$

Dividing this equation by $\theta^{c}$ and letting ' $\theta$ tend to zero we see that the right hand side tends to zero, which implies $\phi(c)=0$ and hence a contradiction.

4. The binomial case. As was mentioned in section 1, the problem of bounded completeness was solved for the binomial case in $[3,4,5]$. Since presumably one is unwilling to estimate the bounded parameter $p$ by means of an unbounded estimate, further work here may seem unnecessary. However, the problem of completeness seems to be of interest for two reasons. If the procedure is bound- 
edly complete without being complete then, even though one may be reluctant to use such an estimate, there may exist an unbounded unbiased estimate of $p$, which for some values of $p$ has smaller variance than the minimum variance bounded estimate. (An example of this is given in [2]). Since this possibility is ruled out when the procedure is complete it is seen that completeness permits statement of a stronger optimum property. Apart from this one may be interested in estimating some unbounded function of $p$ such as $1 / p$. In this case bounded completeness does not permit any statements concerning existence of optimum estimates.

In the present section we shall change our notation somewhat. We are concerned with a sequence of independent trials with constant probability $p$ of success. On the basis of $m$ trials the total number $y$ of successes is a sufficient statistic for $p$. Instead of representing the sufficient statistic for the sequential procedure by $(y, n)$, we shall use the representation $(x, y)$ where $x$ is the total number of failures, so that $x+y=n$. The couples $(x, y)$ may be thought of as making up the points with integral-valued coordinates of the first quadrant of an $x y$-plane, and as before may be classified as boundary points, continuation points, and impossible points. Adopting the terminology of [3], we shall call the value of $x+y$ the index of the point $(x, y)$, so that the points of index $m$ lie on the line $x+y=\mathrm{m}$.

Girshick, Mosteller and Savage defined a sequential procedure to be simple if for each $m$ the continuation points of index $m$ form an interval. They proved that a necessary and sufficient condition for a bounded procedure to be complete is that it be simple. (A procedure is said to be bounded if there exists $N$ so that the number of observations is $\leqq N$.) They also showed that in general simplicity is not sufficient for completeness. However, it was shown later $[4,5]$ that simplicity is sufficient for bounded completeness.

A sequential procedure is said to be closed if the probability of termination is unity for every $p$ with $0<p<1$. It was proved by Girshick, Mosteller and Savage that a necessary condition for completeness of a closed sequential procedure is that no procedure obtained from the given one by removing a boundary point be closed. (Removing a boundary point here means converting it into a continuation point.) We shall prove below that this condition together with simplicity is also sufficient for completeness. An interesting question is whether these two conditions are sufficient for completeness for the general sequential schemes considered in section 2 , when simplicity is replaced by the condition that every procedure obtained from the given one by truncation is complete, and when the second condition is modified by the appropriate null set qualifications. It is easily seen that both of these conditions are necessary.

The following definitions will be needed below. A boundary point $(a, b)$ is a lower (upper) boundary point if for some $x<0(>0)$ the point $(a+x, b-x)$ is a continuation point. An impossible point $(a, b)$ is a lower (upper) impossible point if for some $x<0(>0)$ the point $(a+x, b-x)$ is either a continuation point or a boundary point. 
If the procedure is unbounded every boundary point is either a lower or an upper boundary point. If it is simple, no point can be both an upper and a lower boundary point. The same remarks apply to impossible points.

Theorem. A necessary and sufficient condition for completeness of a closed procedure in the binomial case is that

(i) the procedure is simple, and

(ii) the removal of any boundary point destroys closure.

Proof. Necessity was proved in [3] as was sufficiency for bounded procedures. Sufficiency for unbounded procedures will follow from the following two facts, which we shall prove below.

I. Suppose (i) holds and there exist numbers $a, M>0$ such that for all boundary points $(x, y)$ of index $m \geqq M$ the ratio $y / x \geqq a$. Let $f(x, y)$ be a non-zero unbiased estimate of zero defined over the set $B$ of boundary points, and let $m_{0}$ be the smallest index for which there are points with $f(x, y) \neq 0$. Then $f(x, y)=0$ for all lower boundary points of index $m_{0}$.

II. If (i) holds and if for every positive number $a$ there exist infinitely many boundary points $(x, y)$ with $y / x \leqq a$, then one may remove any lower boundary point without destroying closure.

Suppose now that a sequential procedure satisfies (i) and (ii). Then, since no lower boundary point can be removed without destroying closure, it follows from II. that there exist $a$ and $M$ such that $y / x \geqq a$ for all boundary points of index $\geqq M$. Hence if $f(x, y)$ is an unbiased estimate of zero, and if $m_{0}$ is defined as in I., $f(x, y)=0$ for all lower boundary points of index $m_{0}$. Because of symmetry the statements concerning upper boundary points analogous to I. and II. also hold. It then follows analogously that $f(x, y)=0$ for all upper boundary points of index $m_{0}$. But for a simple unbounded procedure every boundary point is either an upper or a lower boundary point, and hence we obtain a contradiction with the definition of $m_{0}$.

Before proving I. and II. we state the following corollary, which generalises an example given in [3].

Corollary. A sequential procedure that is not bounded and that has a finite non-zero number of lower boundary points is not complete. The analogous result holds for upper boundary points.

Proof of Corollary. This follows easily from II., since if a procedure of this type is to be closed there must exist for each $a>0$ infinitely many upper boundary points $(x, y)$ with $y / x \leqq a$.

In the remainder of the paper we are concerned with the proofs of I. and II.

Proof of I. Assume I. to be false, and let $\left(x_{0}, y_{0}\right)$ be the lowest boundary point of index $m_{0}$ for which $f\left(x_{0}, y_{0}\right) \neq 0$. Then $y>y_{0}$ for all other boundary points $(x, y)$ for which $f(x, y) \neq 0$. Hence if the probability of a point $(x, y)$ is $c(x, y) p^{y} q^{x}$ and if $k(x, y)=c(x, y) f(x, y)$,

$$
k\left(x_{0}, y_{0}\right) p^{y_{0}} q^{x_{0}} \equiv-\Sigma k(x, y) p^{y} q^{x},
$$


where the summation extends over all boundary points of index $\geqq m_{0}$ for which $y>y_{0}$. Dividing both sides by $p^{y_{0}}$ we see that

$$
k\left(x_{0}, y_{0}\right) q^{x_{0}} \equiv-p \Sigma k(x, y) p^{y_{-y_{0}-1}} q^{x} .
$$

If we can show that the expression multiplying $-p$ on the right hand side remains bounded as $p$ tends to zero, we have a contradiction. For letting $p$ tend to zero, we would then see that the right hand side tends to zero and the left hand side to $k\left(x_{0}, y_{0}\right)$, and hence that $f\left(x_{0}, y_{0}\right)=0$.

To prove this, note that

$$
\left|\Sigma k(x, y) p^{y-y_{0}-1} q^{x}\right| \leqq \Sigma|k(x, y)| p^{y-y_{0}-1} .
$$

The right hand side is a power series in $p$. We shall show that this series converges for some $p_{0}>0$. This implies uniform convergence for $|p|<p_{0}$, and therefore the series remains bounded at $p=0$. By assumption there exist numbers $a$ and $M^{\prime}$ such that $y / x \geqq a$ for all boundary points with $y>M^{\prime}$. From now on we shall consider all series as being summed over the set of boundary points for which $y>M^{\prime}$ and hence $q^{x} \geqq q^{y / a}$. Since only a finite number of terms are omitted this does not affect any convergence properties.

Let $0<p_{1}<1$. Then, since $f$ is an unbiased estimate of zero, the series

$$
\Sigma k(x, y) p_{1}^{y} q_{1}^{x}
$$

converges absolutely. Hence, so does

$\Sigma|k(x, y)| p_{1}^{y-y_{0}-1} q_{1}^{x-\frac{1}{a}\left(y_{0}+1\right)} \geqq \Sigma|k(x, y)|\left(q_{1} p_{1}^{\frac{1}{a}}\right)^{y-y_{0}-1}=\Sigma|k(x, y)| p_{0}^{y-y_{0}-1}$, and consequently the last series is convergent.

Proof of II. Let $R$ be any closed simple procedure satisfying the conditions of II., and let $\left(x_{0}, y_{0}\right)$ be any lower boundary point of $R$. We denote by $R^{*}$ the procedure obtained from $R$ by taking $\left(x_{0}, y_{0}\right)$ to be a continuation point and by $n^{*}$ the number of observations for $R^{*}$.

We first prove that any upper impossible point of $R$ is also an impossible point of $R^{*}$. The negation of this would imply that one can get from a lower boundary point to an upper impossible point going only through impossible points. This would require at least one step of either of the following kinds:

Lower impossible point $\rightarrow$ upper impossible point;

Lower boundary point $\rightarrow$ upper impossible point.

One can easily convince oneself with the aid of a diagram that any procedure under which such steps are permitted cannot be simple.

Let $0<p, \pi<1$, and let $a$ be such that $0<a<p / q$. If $p$ is the true probability of success, $y / x$ tends in probability to $p / q$, and hence there exists $N$ such that

$$
P(y / x \geqq a \mid p)>\pi
$$

whenever the index of $(x, y)$ exceeds $N$. By assumption there exists $N_{1}>N$ and a boundary point $\left(x_{1}, y_{1}\right)$ of $R^{*}$ of index $N_{1}$ such that $y_{1} / x_{1} \leqq a$. Then the 
probability exceeds $\pi$ that the random point $(x, y)$ of index $N_{1}$ will lie above $\left(x_{1}, y_{1}\right)$. Since $\left(x_{1}, y_{1}\right)$ is a boundary point, the probability is therefore greater than $\pi$ that the point $(x, y)$ of index $N$ is either an upper impossible point for $R$ and hence impossible for $R^{*}$, or a stopping or continuation point for $R$. We have therefore proved that the probability is $>\pi$ that either $n^{*} \leqq N_{1}$ or the point $(x, y)$ of index $N_{1}$ is a continuation point of $R$.

But given that one has reached a continuation point $(a, b)$ of $R$, there exists $N_{2}$ such that

$$
P\left(n^{*} \leqq N_{2} \mid p,(a, b)\right) \geqq \pi
$$

For

$$
P\left(n^{*}>N_{2} \mid(a, b)\right)=P\left(n>N_{2} \mid(a, b)\right) \rightarrow 0 \text { as } N_{2} \rightarrow \infty .
$$

Since there are only a finite number of continuation points of index $N_{1}$, it is now clear that there exists $N_{0}$ such that

$$
P\left(n^{*} \leqq N_{0} \mid p\right) \geqq \pi+\pi^{2}-1,
$$

which can be made arbitrary close to 1 by proper choice of $\pi$. Therefore $R^{*}$ is closed.

\section{REFERENCES}

[1] E. L. Lehmann and H. Scheffe, "On the problem of similar regions," Proc. Nat. Acad. Sci., Vol. 33 (1947), pp. 382-386.

[2] E. L. Lehmann and H. Scheffe, "Completeness, similar regions and unbiased estimation," unpublished.

[3] M. A. Girshick, Frederick Mosteller and L. J. Savage, "Unbiased estimates for certain binomial sampling problems, with applications," Annals of Math. Stat., Vol. 17 (1946), pp. 13-23.

[4] J. Wolfowitz, "On sequential binomial estimation," Annals of Math. Stat., Vol. 17 (1946), pp. 489-493.

[5] L. J. Savage, "A uniqueness theorem for unbiased sequential binomial estimation," Annals of Math. Stat., Vol. 18 (1947), pp. 295-297.

[6] D. Blackwell, "Conditional expectation and unbiased sequential estimation," $A n$ nals of Math. Stat., Vol. 18 (1947), pp. 105-110. 